\title{
Breve análisis del intercambio comercial de Venezuela y el MERCOSUR: 2006-2015
}

\section{Brief analysis of the trade of Venezuela and MERCOSUR: 2006-2015}

Carolina Lourdes Rodríguez Aguilera

Universidad Simón Bolivar, Venezuela

Autor para correspondencia: rodriguezcarol@usb.ve

Fecha de recepción: 05 de Agosto de 2016 - Fecha de aceptación: 26 de Septiembre de 2016

\begin{abstract}
Resumen
Este trabajo tiene como propósito analizar el comercio de Venezuela y los países del MERCOSUR, entre los años 2006 y 2015, partiendo de la revisión de fuentes nacionales e internacionales en materia estadística, comercial y aduanera. Es un tema de investigación que contribuye al estudio de la integración y pone de relieve una realidad compleja en el escenario económico nacional y regional, donde coexisten intereses nacionales y comunitarios.
\end{abstract}

Palabras claves: integración económica; comercio; mercado común; mercosur

\begin{abstract}
This work aims to analyze trade between Venezuela and the MERCOSUR countries, from 2006 to 2015, based on the review of national and international sources of statistical, trade, and customs matters. It is a research topic that contributes to the study of integration and highlights a complex reality in the national and regional economic environment where coexist national and community interests.
\end{abstract}

Key words: economic integration; trade; common market; mercosur 


\section{Introducción}

El Tratado de Asunción (1991) enfatiza la necesidad de alcanzar el objetivo final de un mercado común con base en los principios de gradualidad, flexibilidad y equilibrio. Estos principios, permiten explicar los tratamientos favorables entre los países miembros, y la adaptación gradual según sus necesidades y particularidades, en especial los países de menor grado de desarrollo.

Con ese marco referencial, es pertinente señalar que el proceso de incorporación de Venezuela como Estado Parte del MERCOSUR se inició en Montevideo, con la firma del Acuerdo Marco para la adhesión de Venezuela al MERCOSUR, el 9 de diciembre de 2005. Luego se concretó la suscripción del Protocolo de Adhesión, en Caracas el 4 de julio de 2006, y finalmente, la aprobación del Protocolo de Adhesión de Venezuela al MERCOSUR en Brasilia, el 12 de agosto de 2012, que formalizó el ingreso como Estado Parte en esa organización de integración regional intergubernamental.

En ese orden de ideas, este trabajo propone ilustrar el intercambio comercial de Venezuela con los países del MERCOSUR, entre los años 2006 y 2015. Es preciso anotar que se consultaron diversas fuentes tanto nacionales como internacionales: como el Instituto Nacional de Estadística de Venezuela (INE), el Banco Central de Venezuela (BCV), el Servicio Nacional Integrado de Administración Aduanera y Tributaria (SENIAT), el Centro de Comercio Internacional Trade Map, y la Urunet MERCOSUR.

Asimismo, se realizará un análisis cuantitativo y cualitativo de la contribución de las exportaciones e importaciones de Venezuela en el MERCOSUR.

Ahora bien, cuando se intenta abordar la integración económica, puede entendérsela como un proceso a través del cual, dos o más mercados nacionales se vinculan para formar un mercado mayor, en este orden de ideas, hay autores ${ }^{1}$ que coinciden en esta percepción. En palabras de Ellsworth y Leith (2004) "la integración económica es la unificación de distintas economías en una sola más grande" 2 .

Dicho enfoque supone varios puntos a tomar en cuenta, por ejemplo, exige la realización de una serie de tareas y actividades para el logro de determinados objetivos, los cuales dependerán de la voluntad originaria de los Estados, que se vinculan con otros, a través de la suscripción de un acuerdo internacional.

En principio, uno de los objetivos básicos de la integración económica es la eliminación de barreras arancelarias y administrativas que obstaculizan los intercambios comerciales recíprocos, aunque en procesos avanzados ${ }^{3}$ operan objetivos más amplios como pueden ser, la armonización de políticas económicas y sociales, y la coordinación de planes de desarrollo, o la

\footnotetext{
${ }^{1}$ Como Grien Raúl, Robertson, David, y Tamamés Ramón, Ellsworth y Leith. Testimonio comunitario, 61-62.

${ }^{2}$ Ibídem.566.

${ }^{3}$ La Unión Europea es el referente inmediato.
} 
planificación conjunta. De esta forma, la integración regional puede tener dimensiones distintas ${ }^{4}$, que puede trazarse como metas la constitución de una zona de libre comercio, unión aduanera, o unión económica y monetaria 5 .

Debe señalarse que el MERCOSUR es un modelo de integración destinado a alcanzar un mercado común, con la finalidad de lograr el desarrollo económico y social de los países comprometidos en dicho proceso, según lo acordado en el Tratado de Asunción (1991).

\section{Análisis del Intercambio Comercial entre Venezuela-Mercosur: 2006 - 2014}

En este apartado se abordará el intercambio comercial desde el sector no petrolero de la economía nacional. En primer término, se precisará la información de la participación del comercio de Venezuela en el MERCOSUR en el período 2006-2014.

En ese sentido, cabe advertir que Venezuela registra un intercambio comercial con el MERCOSUR que se ubica en promedio en 6.032 millones de dólares (USD) anuales para el período 2006 - 2014. De ese monto, las exportaciones se sitúan en USD. 225 MM lo que representa cerca del 4\%, mientras que las importaciones representan en promedio USD 5.806 MM, que significa $96 \%$ del comercio, constituyendo una balanza comercial desfavorable para Venezuela, como puede verse en el Gráfico $\mathrm{N}^{\circ} 1$ y Gráfico $\mathrm{N}^{\circ} 2$.

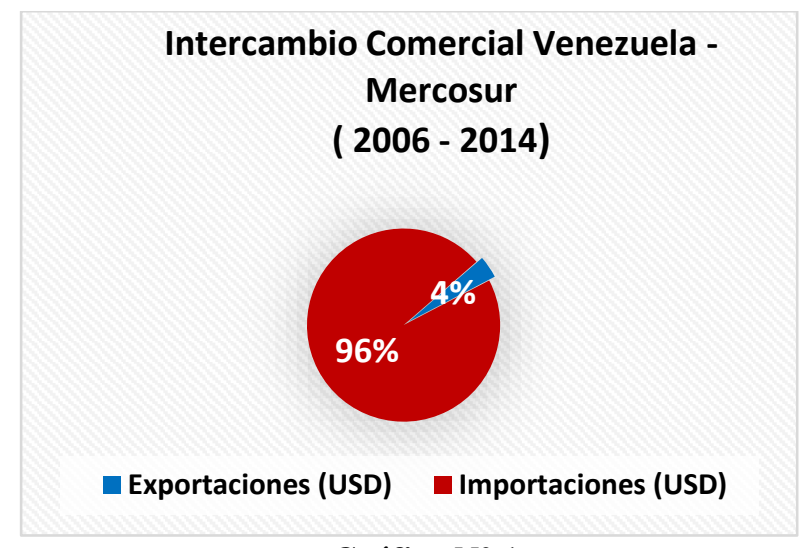

Gráfico $\mathbf{N}^{\circ} 1$

Fuente: Instituto Nacional de Estadística (INE) Elaboración propia

\footnotetext{
${ }^{4}$ Incluso la integración puede abordarse desde distintas concepciones ideológicas y etapas del proceso. La integración económica puede estudiarse desde varios enfoques, liberal, estructural y dirigista; visiones de la integración económica, como proceso y como hecho. Como proceso, destacan las medidas dirigidas a la eliminación para llegar al hecho final. Como hecho, se refiere al objetivo final que se pretende alcanzar luego de eliminar la discriminación entre economías nacionales. Obaya Martín. ¿Qué puede decir la nueva economía institucional sobre los procesos de integración regional? Perspectivas Internacionales, 1, 15-18.

${ }^{5}$ De manera breve, puede explicarse que la zona de libre comercio constituye un área por donde circulan libremente las mercaderías, sin pagar arancel y sin restricciones, pero los países mantienen autonomía de su política comercial con terceros países. En la Unión Aduanera, existe libre comercio entre un grupo de países y éstos aplican un arancel externo común para las importaciones procedentes de terceros países. Por su parte, el mercado común busca además de la libre circulación de bienes y servicios, movilidad de factores (capital, trabajo). Por su parte, la unión económica involucra metas adicionales como armonización de sus políticas económicas nacionales; y, la unión monetaria suma una política monetaria común. Mattli, Walter. The logic of Regional Integration.111-112.
} 
Intercambio comercial Venezuela-Mercosur 2006 - 2014 Expresado en dólares americanos

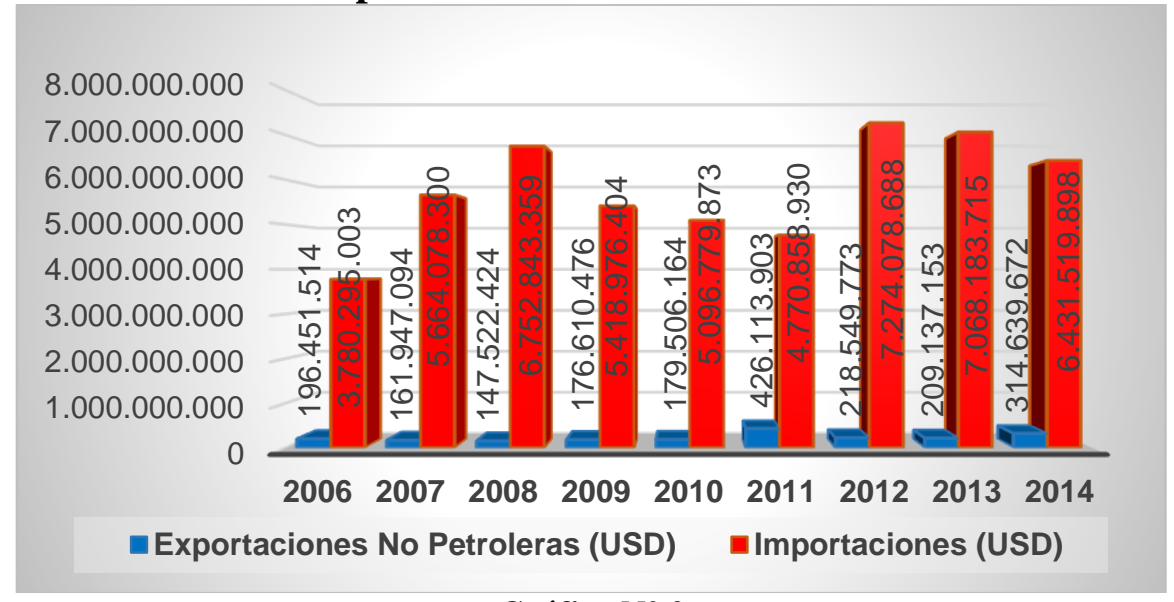

Gráfico $\mathbf{N}^{\circ} 2$

Fuente: Instituto Nacional de Estadística (INE)

Elaboración propia

Para Venezuela es un objetivo primordial potenciar el comercio no petrolero de manera de diversificar la producción nacional y equilibrar la economía nacional. Además, la situación actual exige el aumento de la participación comercial de Venezuela en el bloque regional, y es fundamental la generación de cadenas productivas entre los países del MERCOSUR para generar una verdadera y profunda integración.

Ahora bien, cuando se investiga los principales destinos de las exportaciones no petroleras venezolanas en el MERCOSUR, se advierte que Brasil es el primer mercado, captando el 92\% de las exportaciones venezolanas; en segundo lugar, aparece Argentina con un 6\%; en tercer lugar Uruguay con $2 \%$ y finalmente Paraguay con $0,2 \%$. Véase Gráfico $\mathrm{N}^{\circ} 3$.

\section{Principales destinos de las exportaciones venezolanas hacia Mercosur ( 2006 - 2014)}

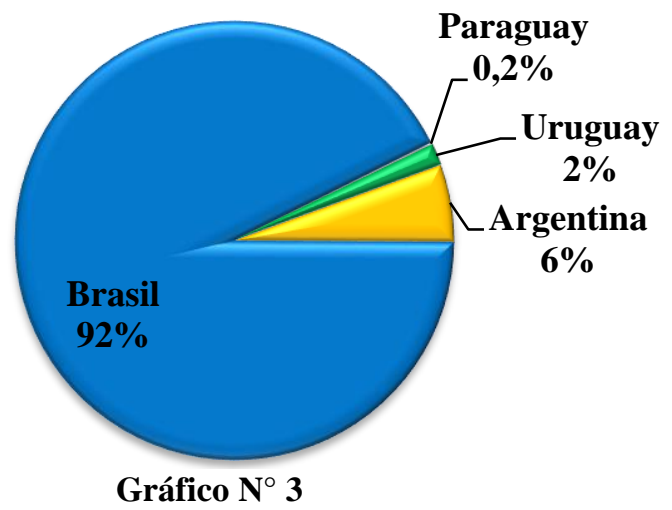

Fuente: Instituto Nacional de Estadística (INE)

Elaboración propia 
Adicionalmente, al revisar la procedencia de las importaciones desde el MERCOSUR en el período 2006-2014, se observa coincidentemente que Brasil el principal país proveedor de bienes para Venezuela con 69\%; en segundo lugar, Argentina con un 24\%; en tercer lugar Uruguay con 6\%; y finalmente Paraguay con $1 \%$. Véase Gráfico № 4.

\section{Principales países proveedores de Venezuela originarios del Mercosur (Período 2006 - 2014)}

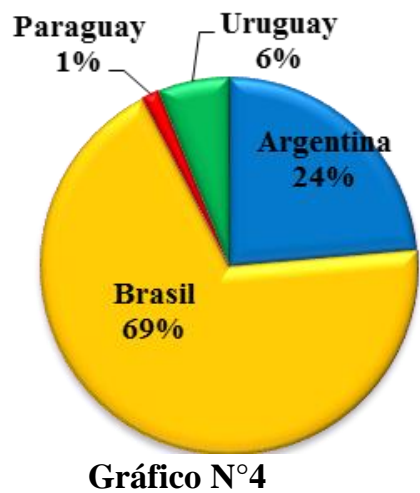

Fuente: Instituto Nacional de estadística (INE)

Elaboración propia

También conviene señalar que cerca del $90 \%$ de las exportaciones no petroleras de Venezuela, son realizadas por las empresas del Estado. Como por ejemplo, Fertilizantes Nitrogenados de Venezuela, Petroquímica de Venezuela, Corporación Eléctrica Nacional (CORPOELEC), Metanol de Oriente (METOR), SIDOR C.A, Supermetanol Carbones de la Guajira Productos de Alcoholes hidratados, Alambres y cables venezolanos ( INE, TRADE MAP, 2016).

Por otra parte, dentro de las empresas mixtas que representa la fusión del capital estatal y capital privado, destacan sociedades que han desarrollado su actividad a partir de las ventajas competitivas que otorga la petroquímica y las industrias básicas, como: PRALCA, Productora de Alcoholes Hidratados, C.A., METANOL DE ORIENTE, Productos de acero Lamigal C.A., Industrias químicas Tripoliven, Industria Nacional Fabrica de Radiadores (Infra, S.A.), Sural C.A., Guardian de Venezuela. Estas empresas concentran prácticamente la mitad de las exportaciones de las empresas privadas que participan del comercio con el MERCOSUR. El resto de las exportaciones venezolanas al MERCOSUR quedan atomizadas cerca de un centenar de pequeñas empresas que en su mayoría realizan labor de comercialización en lugar de producción (INE, 2016). Véase el Gráfico $\mathrm{N}^{\circ} 5$ y Nº 6 . 


\section{Exportaciones No Petroleras venezolanas hacia el MERCOSUR 2006 - 2014 \\ (Expresado en Millones de USD)}

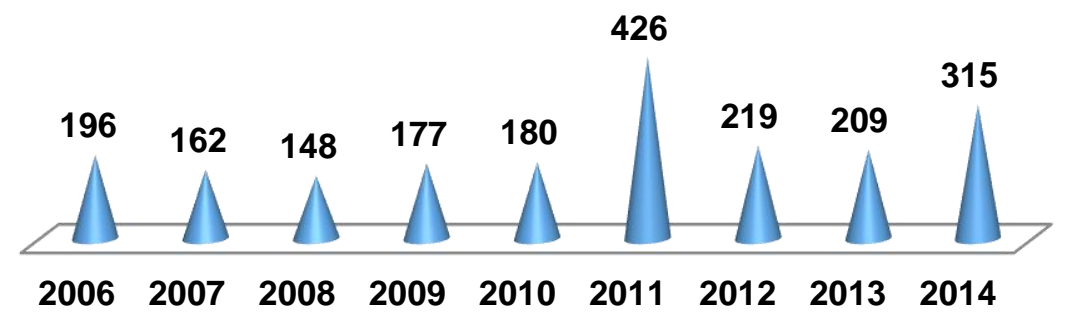

Gráfico $\mathbf{N}^{\circ}$

Fuente: Instituto Nacional de Estadística (INE)

Elaboración propia

Exportaciones Totales No petroleras venezolanas hacia el MERCOSUR, por Sector : 2006 - 2014 (Expresados en USD)

\begin{tabular}{|c|c|}
\hline Productos Químicos & 529.040 .973 \\
\hline Productos Minerales & 645.708 .633 \\
\hline Plástico y sus Manufacturas & 65.957.121 \\
\hline Pieles, Cueros y manufacturas & 440.205 \\
\hline Piedra, Cerámica, Yeso, Vidrio y.. & 43.102 .477 \\
\hline Papel y Cartón & 1.130 .785 \\
\hline Metales Comunes y sus manufacturas & 605.379 .076 \\
\hline Materias Textiles, Calzado y Manufacturas & 2.619 .612 \\
\hline Material Eléctrico & 45.366 .334 \\
\hline Material de Transporte & 69.980 .369 \\
\hline Manufacturas Diversas & 17.152 .163 \\
\hline Madera, Corcho, Carbón Vegetal y.. & 2.062 .439 \\
\hline Industrias Alimenticias & 1.150 .714 \\
\hline Bebidas y Tabaco & 656.605 \\
\hline Agrícola y Vegetal & 730.667 \\
\hline
\end{tabular}

Gráfico $\mathbf{N}^{\circ} 6$

Fuente: Instituto Nacional de Estadística (INE)

Elaboración propia

Es pertinente señalar que las exportaciones no petroleras venezolanas hacia Argentina se concentran en los siguientes productos: Abonos (77\%), metanol (5\%), Máquinas de perforación (2\%), Dietilenglicol (2\%).

Mientras que, las exportaciones no petroleras venezolanas hacia Brasil se concentran en los siguientes productos: Naftaleno (45\%), Metanol (19\%), Energía Eléctrica (6\%), productos laminados de hierro o acero (4\%), Etilenglicol (3\%) y Hulla bituminosa (1\%). 
Por otra parte, las exportaciones no petroleras venezolanas hacia Paraguay comprenden los siguientes productos: Pinturas (27\%), productos plásticos (19\%), Colorantes pigmentarios $(18 \%)$, Etiquetas $(7 \%)$, Poliamidas en formas primarias $(6 \%)$, las demás resinas $(6 \%)$, preparaciones tensoactivas para lavar $(4 \%)$.

Finalmente, las exportaciones no petroleras venezolanas hacia Uruguay abarcan productos como: Abonos minerales (87\%), Insecticidas (3\%), Etilenglicol (3\%), Trifosfato de sodio $(2 \%)$ y Medicinas $(2 \%)$.

Debe precisarse que el número de exportadores venezolanos hacia el MERCOSUR ha mermado considerablemente en el período seleccionado, las exigencias y controles administrativos y burocráticos y la inestabilidad de la economía nacional, son causas profundas que explican esa tendencia. Véase Gráfico $\mathrm{N}^{\circ} 7$.

\section{Número de exportadores venezolanos hacia el MERCOSUR $2006-2014$}

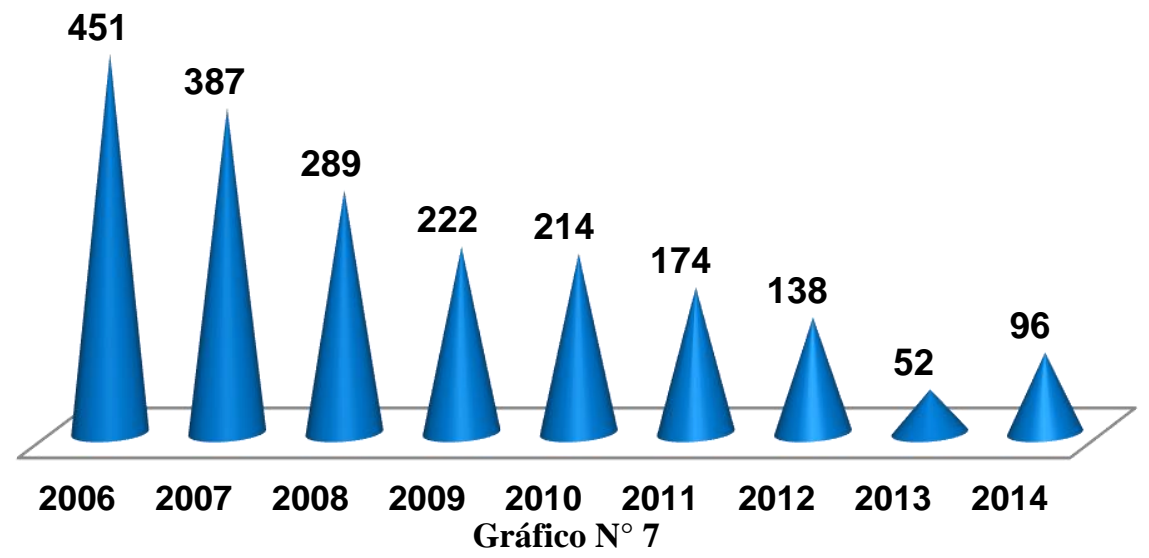

Fuente: Instituto Nacional de Estadística (INE)

Elaboración propia

\section{Intercambio Comercial Venezuela-Mercosur: enero-septiembre 2015}

Ahora bien, partiendo de la revisión de las exportaciones de los países del MERCOSUR con destino a Venezuela en el período enero-septiembre de $2015^{6}$, se puede observar que Brasil es el principal país proveedor de bienes para Venezuela, representando una participación de 62,56\%, que constituye 2.303.436.880 USD del mercado del total de las importaciones que realiza Venezuela procedentes del MERCOSUR. Argentina se ubica en el segundo lugar con una participación de 32,86\%, esto es 1.209.850.606 USD; en tercer lugar está Uruguay que representa 3,86\% de las importaciones sureñas, que se traduce en 142.293.774 USD; y en último lugar, están las importaciones originarias de Paraguay que constituyen el 0,72\% esto representan 26.540.105 USD. Véase Cuadro $\mathrm{N}^{\circ} 1$ y Gráfico $\mathrm{N}^{\circ} 8$.

\footnotetext{
${ }^{6}$ Debe acotarse que las estadísticas disponibles en las fuentes oficiales consultadas como Banco Central de Venezuela e Instituto Nacional de Estadísticas sólo contiene información hasta el tercer trimestre de 2015.
} 


\begin{tabular}{lll}
\hline \multicolumn{2}{l}{ Exportaciones de los Países del MERCOSUR con destino a Venezuela } \\
\hline \multicolumn{2}{l}{ enero - septiembre de $\mathbf{2 0 1 5}$} & Participación \% \\
$\begin{array}{l}\text { (Expresados en USD) } \\
\text { País Exportador }\end{array}$ & Total & \\
& & $32,86 \%$ \\
Argentina & 1.209 .850 .606 & $62,56 \%$ \\
Brasil & 2.303 .436 .880 & $0,72 \%$ \\
Paraguay & 26.540 .105 & $3,86 \%$ \\
Uruguay & 142.293 .774 & $\mathbf{1 0 0 \%}$ \\
Total general & $\mathbf{3 . 6 8 2 . 1 2 1 . 3 6 5}$ \\
& Cuadro $\mathbf{N}^{\circ} \mathbf{1}$ \\
\hline & Fuente: Urunet \\
& Elaboración propia
\end{tabular}

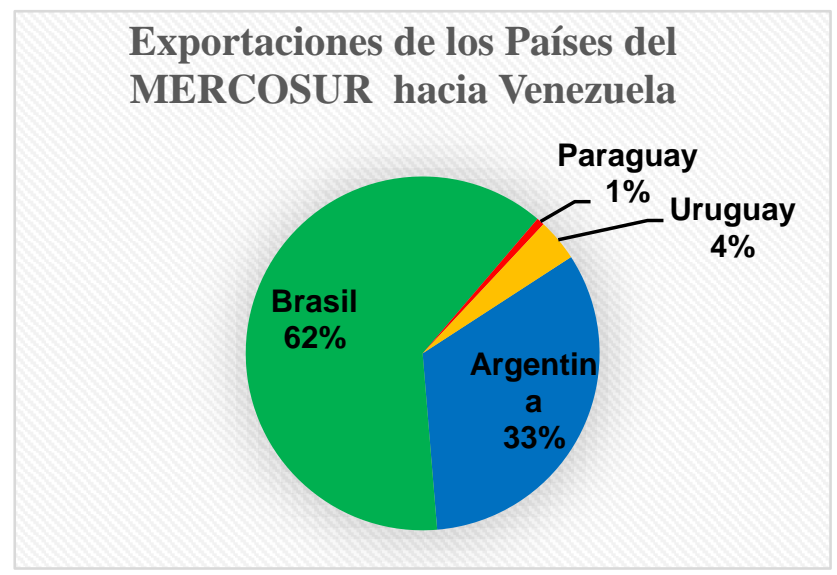

Gráfico $\mathbf{N}^{\circ} 8$

Fuente: Urunet

Elaboración propia

Asimismo, resumiendo las exportaciones totales procedentes de MERCOSUR hacia Venezuela para el período analizado de enero-septiembre 2015 expresados en USD, pueden agruparse por los siguientes sectores representativos. Véase Cuadro $\mathrm{N}^{\circ} 2$.

\begin{tabular}{lllll}
\hline Sector & & Total & & Participación \% \\
\cline { 2 - 2 } Agrícola y Vegetal & & $1,870,735,133$ & & $50.8 \%$ \\
Bebidas y Tabaco & & $5,398,796$ & & $0.1 \%$ \\
Industrias Alimenticias & & $634,108,767$ & & $17.2 \%$ \\
Madera, Corcho, Carbón Vegetal y Manufacturas & 252,011 & & $0.0 \%$ \\
Manufacturas Diversas & & $365,917,586$ & & $9.9 \%$ \\
Material de Transporte & & $58,410,095$ & & $1.6 \%$ \\
Material Eléctrico & $273,796,978$ & & $7.4 \%$ \\
Materias Textiles, Calzado y Manufacturas & & $19,912,549$ & & $0.5 \%$ \\
Metales Comunes y sus manufacturas & & $85,735,142$ & & $2.3 \%$ \\
Papel y Cartón & $45,309,707$ & & $1.2 \%$ \\
\hline
\end{tabular}




\begin{tabular}{lll}
\hline Piedra, Cerámica, Yeso, Vidrio y Manufacturas & $4,083,054$ & $0.1 \%$ \\
Pieles, Cueros y manufacturas & 303,808 & $0.0 \%$ \\
Plástico y sus Manufacturas & $70,762,421$ & $1.9 \%$ \\
Productos Minerales & 22,574 & $0.0 \%$ \\
Productos Químicos & $247,372,744$ & $6.7 \%$ \\
Total general & $\mathbf{3 , 6 8 2 , 1 2 1 , 3 6 5}$ & $\mathbf{1 0 0 \%}$ \\
\hline
\end{tabular}

Cuadro $\mathbf{N}^{\circ} 2$

Fuente: Urunet

Elaboración propia

Por otra parte, el examen de las importaciones de los países del MERCOSUR provenientes de Venezuela para el período enero-septiembre de 2015, denota que Brasil se mantiene como el principal mercado de las exportaciones venezolanas, representando el 78,10\% del mercado regional. Esto se traduce en 562.680.218 USD. La segunda posición la ocupa Uruguay con el 21,37\% esto representa 153.994.366 USD. Argentina pasó a un tercer lugar como destino de las exportaciones venezolanas con el 0,32\% que constituye 2.288.651 USD. Y finalmente se encuentra Paraguay con una participación de 0,21\% esto es 1.508.115 USD. Véase Cuadro $\mathrm{N}^{\circ} 3$ y Gráfico $\mathrm{N}^{\circ} 9$.

\begin{tabular}{|c|c|c|}
\hline \multicolumn{3}{|c|}{ Importaciones de los Países del MERCOSUR provenientes de Venezuela } \\
\hline \multicolumn{3}{|c|}{ enero - septiembre de 2015} \\
\hline \multicolumn{3}{|c|}{ (Expresados en USD) } \\
\hline País Importador & Total & Participación \% \\
\hline Argentina & 2.288 .651 & $0,32 \%$ \\
\hline Brasil & 562.680 .218 & $78,10 \%$ \\
\hline Paraguay & 1.508 .115 & $0,21 \%$ \\
\hline Uruguay & 153.994.366 & $21,37 \%$ \\
\hline Total general & 720.471.349 & $100,00 \%$ \\
\hline
\end{tabular}

Cuadro $\mathbf{N}^{\circ} 3$

Fuente: Urunet

Elaboración propia

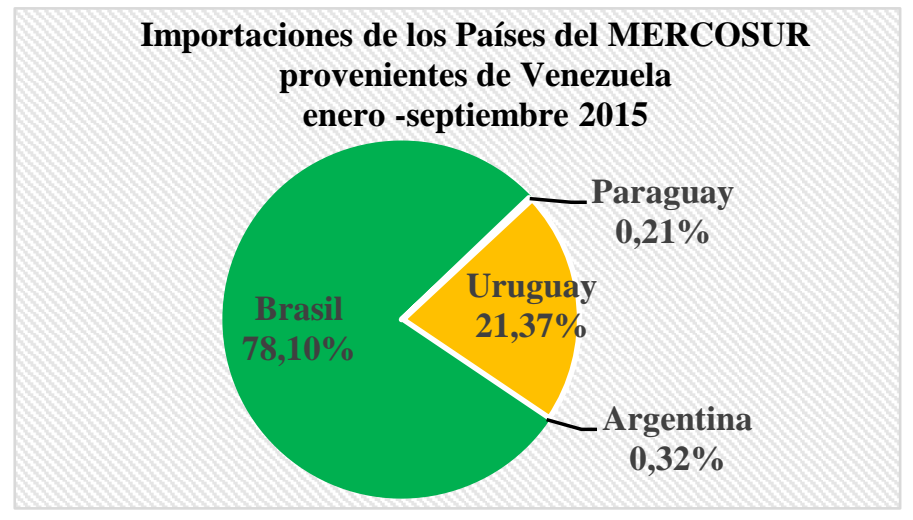

Gráfico $\mathbf{N}^{\circ} 9$

Fuente: Urunet

Elaboración propia 
En cuanto, a las exportaciones venezolanas hacia Argentina durante el período enero septiembre de 2015, presentaron una caída de 65,93\% durante el período enero-septiembre 2014/2015 al pasar de 7,7 millones de USD a 2,7 millones 653,010.91, la caída de USD 5,1 millones, al no exportar el Coque (2,6 Millones de USD), Urea en 2,3 millones de USD y la disminución de las ventas de ferrolicio en 1,1 millones de USD. Los principales productos exportados: etanodiol (23\%), tubos de hierro (16\%), ferrosilicio $(12 \%)$ y radiadores para vehículos $(8 \%)$ y el carbono negro de humo (4\%).

Cabe resaltar que las exportaciones venezolanas se concentran en Brasil (78\%) destacándose como principales productos $(91 \%)$ : Naftas para petroquímica $(60,1 \%)$, Coque de petróleo (10,9\%), Metanol (7,1\%), Energía eléctrica (5,5\%), Hulla bituminosa, no aglomerada $(4,5 \%)$, y Urea con un contenido de nitrógeno $>45 \%$ en peso $(2.8 \%)$.

Por otra parte, las importaciones que ha realizado Paraguay desde Venezuela, entre enero y septiembre de 2015, abarcan los siguientes productos: el coque, betún de petróleo $(96,6 \%)$, correas para transmisión $(0,64 \%)$, resinas alcidicas $(0.6 \%)$, aparatos fabricación pastas alimenticias y maquinas $(0.5 \%)$, y aparatos para usos sanitarios $(0,4 \%)$.

Mientras que, las importaciones que realizó Uruguay desde Venezuela, entre enero y septiembre de 2015, tuvieron la siguiente composición sectorial: leche (47\%), arroz (18\%), quesos de pasta semidura (9\%), y neumáticos de autobuses (5\%). En el período estudiado de 2015, se observa una caída de las ventas de carne bobino, con respecto al mismo período del 2014. De manera que, en el 2014 representaron 44 millones de USD, y en el 2015, registraron 1,9 millones de USD. Asimismo, disminuyeron las ventas de leche, quesos y mantequilla.

\section{Conclusiones}

El proceso de integración económica regional implica la vinculación de sistemas nacionales e institucionales distintos, y exige la activación de la producción y de las relaciones entre los sectores productivos nacionales que forman parte de ese mercado ampliado. Además, requiere resultados para las economías de los países participantes, para las industrias y un mejoramiento del nivel de vida de la población.

Consecuentemente con lo expuesto, se hace necesario realizar un esfuerzo decidido y mancomunado entre los sectores públicos y privados para diversificar los actores económicos presentes en el comercio con el MERCOSUR.

La participación en un grupo de integración regional involucra el intercambio de mercancías y servicios; así como, la relación comercial del bloque con terceros países (arancel externo común), entre otras acciones, que representan espacios de cooperación y fomento de la vecindad entre los países.

Debe reconocerse que, las realidades nacionales de los países, las tensiones políticas y las demandas sociales, representan un factor de preocupación en el camino de la integración regional y en su consolidación. 
El intercambio comercial de Venezuela con los países del MERCOSUR, en los sectores no petroleros, muestran un balance desfavorable para Venezuela, que exige la puesta en práctica de políticas públicas que impulsen y diversifiquen la producción nacional, así como, la promoción de las exportaciones.

Es imperioso procurar el desarrollo nacional fundamentando en la capacidad de los países de participar en la creación y difusión de conocimientos y tecnologías y su incorporación en la actividad económica. Fomentar la capacidad de gestión y organización de los recursos disponibles, aumentar la producción, y el empleo. Gestionando que las exportaciones ofrezcan un valor agregado que potencie las oportunidades para el país. En ese orden, la integración regional brinda oportunidades como la ampliación de mercados y facilita el acceso a las inversiones y las tecnologías.

Asimismo, es preciso diseñar programas de financiamientos especiales que incentiven la producción y exportación de bienes y servicios, así como, promover y diversificar la oferta exportable y una cultura exportadora en Venezuela.

\section{Bibliografía}

Banco Central de Venezuela (2016). Información Estadística del BCV. http://www.bcv.org.ve/c2/indicadores.asp . [Consulta: 2016, junio 18].

Centro de Comercio Internacional Trade Map (2016). http://www.trademap.org/Index.aspx. [Consulta: 2016, mayo 06].

Comisión Presidencial (2005). Acuerdo Marco para la Adhesión de Venezuela al Mercosur (9 de diciembre de 2005). Montevideo.

Comisión Presidencial (2006). Protocolo de Adhesión de la República Bolivariana de Venezuela. (4 de julio de 2006). Caracas.

Consejo del Mercado Común (1991). Tratado de Asunción. http://www.mercosur.int/innovaportal/v/5271/2/innova.front/tratados-protocolos-yacuerdos. [Consulta: 2016, mayo 23].

Instituto Nacional de Estadística de Venezuela (2016). http://www.ine.gov.ve/ http://www.ine.gov.ve/index.php?option=com_content\&view=category\&id=48\&Itemid= 33. [Consulta: 2016, junio 30].

Mattli, Walter. (2001). The logic of Regional Integration. United Kingdom. Cambridge: University Press.

Obaya Martín. (2005)¿Qué puede decir la nueva economía institucional sobre los procesos de integración regional? Perspectivas Internacionales, 1, 15-18. 
Servicio Nacional Integrado de Administración Aduanera y Tributaria (SENIAT) (2016). http://declaraciones.seniat.gob.ve/portal/page/portal/Manejador_Contenido_SenIAT/02N ormativa_Legal.html. [Consulta: 2016, abril 07].

SENIAT (2016). Aduanas. http://declaraciones.seniat.gob.ve/portal/page/portal/Manejador_Contenido_Seniat/04adu anas/index.html . [Consulta: 2016, abril 09].

Tribunal de Justicia de la Comunidad Andina (2004). Testimonio Comunitario. Quito: TJCA, 6162.

Urunet MERCOSUR (2016) Base de datos de Comercio Exterior. http://urunetmercosuronline.com/. [Consulta: 2016, mayo 28]. 